NOTE

\title{
SUR L'EFFET DES CORPS FLOTTANTS SUR L'ÉCOULEMENT DES RIVIĖRES ET POUSSÉE RÉSULTANTE SUR LES BARRAGES
}

\author{
L. VADOT \\ Ingénieur-Docteur de l'Université de Nancy
}

Les rivières et canaux dont l'écoulement s'effectue le plus généralement avec surface libre, peuvent, dans certains cas particuliers, présenter une surface encombrée par les corps flottants. Il s'agira le pluts fréquemment de trones d'arbres (flottage) ou de glace. C'est surtout ce dernier aspect qui retiendra jotre attention, le flottage ne présentant plus, en Europe, l'importance qu'il avait autrefois.

Lorsque la température s'abaisse de facon suffsante, et que la durée du froid est assez grande, une certaine partic de l'eau des rivières peut se prendre en glace. Généralement, le gel fait d'abord sentir son action sur les rives où les vitesses sont plus faibles. Des morceaux de glace s'en détachent, et sont charriess par Je courant. Si le froid est suffisamment. intense, la quantité de glace ainsi véhiculée augmente, et moyennant certaines conditions locales (rétrécissement du lit, obstacle artificiel, piles de ponts, (tc..), un embouteillage complet peut se produire. Les blocs se coïncent les uns contre les autres (embâcle), formant à la surface de l'eau une croûte solide « relativement » fixe. Les blocs étant arrêtés ont tendance à se souder facilement. Le barrage flottant ainsi constitué arrête les blocs charriés, et la couche de glace s'accroît en dimension. Sous l'effet du courant, les blocs s'accumulent à l'aval ou se glissent sous la croûte superficielle, l'épaisseur de glace augment’, et une obstruction quasi-complète du lit arrivera même, entrainant les accidents que l'on imagine.

Nous ne nous étendrons pas, ici, sur le processus de formation de la glace, problème qui ne nous intéresse pas directement. Nous dirons toutefois quelques mots des différentes sortes de glace que l'on rencontre. Outre les morceanx détachés des ríves, le courant peut charrier des blocs ayant une autre provenance. De petits morceaux de glace (une véritable suspension colloüdale de germes de glase peut même exister dans l'eau) et la neige tombant dans l'eau peuvent devenir des noyaux de cristallisation, et donner naissance à des blocs importants dont la forme est plus régulière que celle des blocs délachés des rives. Une autre forme peut apparaître, quoique plus rarement, e'est la glace de fond. Si la turbulence de l'eau est grande, ainsi que l'agitation de surface, par suite du contact intime avec l'atmosphère et des occlusions d'air frais, une forte baisse de température peut se transmettre jusqu'au fond du lit où la vitesse plus faible favorise la formation d'une couche glacéc adhérente au sol ; la croissancer de cette couche s'effectuera de bas en haut et pent même aller jusqu'à l'apparition de véritables îlots de glace (observé en Russie). Ce genre de glace lié à l'existence d'une turbulence de toute la masse apparaîtra en particulier à la suite d'un troncon tumultueux. Sa formation sera entravée par une couche de glace superficielle qui empêche l'apport de froid de l'atmosphère au fond de la rivière.

\section{Influence de la couche de glace sur le niveau d'eau}

Voyons, maintenant, quel peut être l'effet d'une couche de glace superficielle sur l'écoulement d'une. rivière : 
Soit B la largeur de la rivière, h la profondeur, i la pente du radier. Considérons un tronçon de courant se trouvant dans la position 1 avec les caractéristiques : $\mathrm{B}_{1}, \mathrm{~h}_{1}, \mathbf{i}_{1}$, celui-ci a sa surface animée d'une vitesse moye nne $V_{1}$. L'encombrement des glaces est déterminé par le paramètre $\sigma_{1}$ : rapport de de la surface des glacons a la surface libre totale de ce tronçon. Un certain temps après, le tronçon envisagé se trouve dans la position 2 caractérisée par: $\mathrm{B}_{2}, \mathbf{h}_{2}$. $\mathbf{i}_{2}, \mathrm{~V}_{2}$, et $\sigma_{2}$. Les valeurs $\sigma_{1}$ et $\sigma_{2}$ de $\sigma$ sont comprises entre 0 (surface sans glace) et 1 (sura face entièrement prise).
Considérons, par exemple, deux sections différentes :

$\mathrm{B}_{1}=300 \mathrm{~m} . \quad \mathrm{h}_{1}=3 \mathrm{~m} .80 \quad \mathrm{i}_{1}=\mathrm{i}_{2} \quad \mathrm{C}_{1}=\mathrm{C}_{2}$ $\mathrm{B}_{2}=100 \mathrm{~m}$. $\quad \mathrm{h}_{2}=8 \mathrm{~m}$.

En première approximation, nous avons admis l'égalité des coefficients $\mathrm{C}_{1}$ et $\mathrm{C}_{2}$ en faisant toutefois remarquer, qu'en toute rigueur, cette hypothèse ne serait applicable qu'à la condition d'admettre $\sigma_{1}$ peu différent de $\sigma_{2}$

Si dans la section 1 on observe une quantité de glace telle que $\sigma=0,5$, on obtiendra pour la section 2 ,

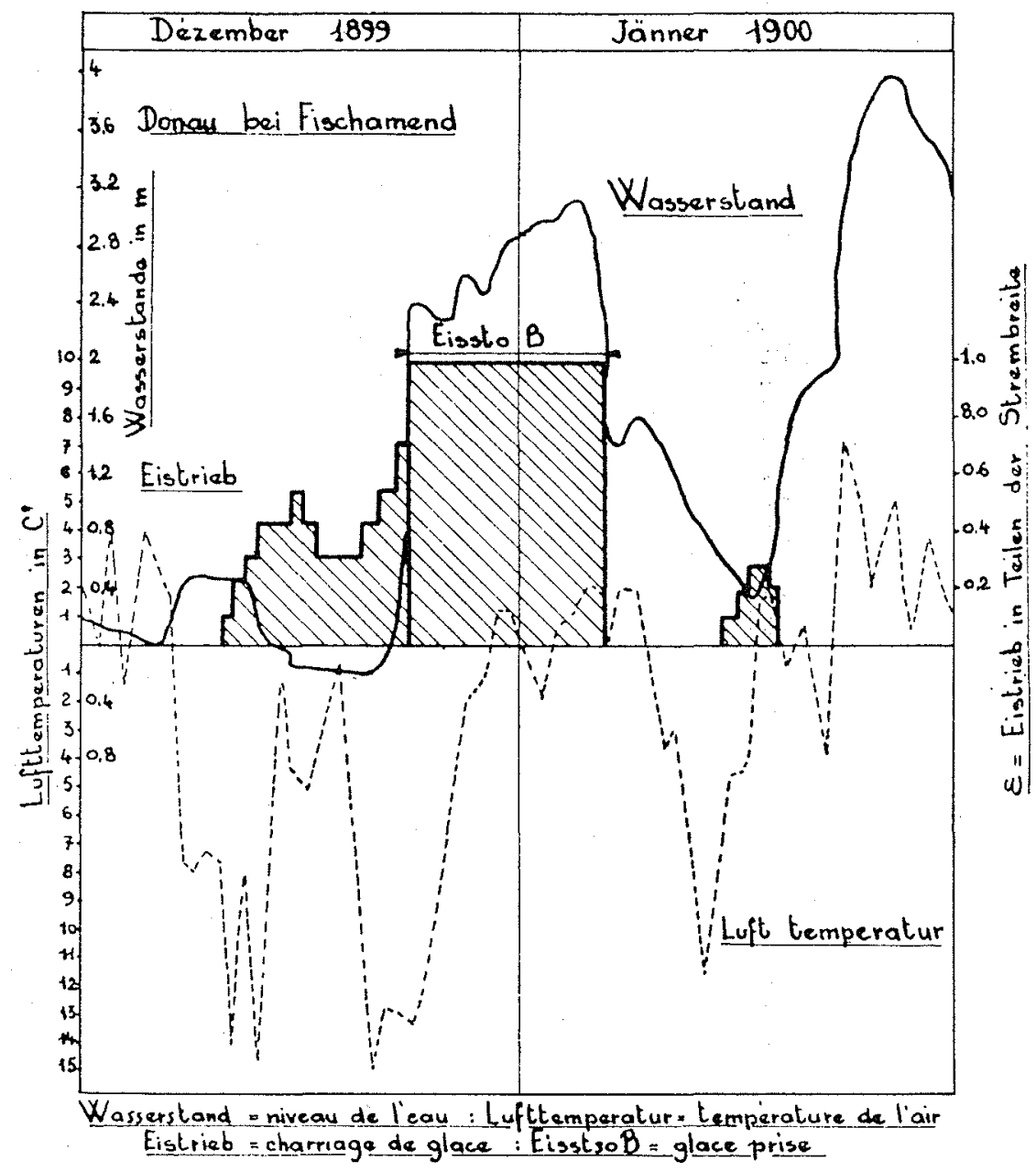

FIG. 1

Il est aisé de voir que $\sigma, B$ et $V$ sont liés par la relation suivante : $\sigma_{1} B_{1} V_{1}=\sigma_{2} B_{2} V_{2}$ Posons : d'où

$$
V=C \sqrt{R_{h} i} \text { avec } R_{h}=h \text { rayon hydraulique }
$$

$$
\sigma_{2}=\sigma_{1} \frac{C_{1}}{C_{2}} \frac{B_{1}}{B_{1}} \sqrt{\frac{h_{1}}{h_{2}} \frac{i_{1}}{i_{2}}}
$$

$\tau_{2}=1,04$. Ceci signifie que les glaçons sont pris dans une section 1', en amont de la section 2. La couche superficielle étant complètement formée à partir de la section 1', l'écoulement s'effectue alors à travers un contour fermé.

Supposons la rivière assez large, afin de pouvoir négliger l'effet des rives dans une même section, le 
rayon hydraulique prend les valeurs ci-après, suivant les deux régimes:

$$
\begin{aligned}
& \text { a surface libre } R_{\mathrm{h}}=\mathrm{h} \\
& \text { à surface prise } R_{\mathrm{h} g}=\frac{\mathrm{hg}}{2}
\end{aligned}
$$

Le débit par unité de largeur, pour les deux régimes est respectivement :

$$
\begin{aligned}
& \text { à surface libre } q=h C \sqrt{h \dot{i}} \\
& \text { à surface prise } q_{g}=h_{g} C^{\prime} \sqrt{\frac{h g}{2}}
\end{aligned}
$$

Dès lors, si le débit est le même $\left(q_{\mathrm{I}}=q_{\mathrm{g}}\right)$ et dans l'hypothèse où la rugosité de la croûte de glace est égale à celle des parois $\left(\mathrm{C}=\mathrm{C}^{\prime}\right)$, h et hg sont liés par la relation suivante:

$$
\begin{gathered}
\lg \sqrt{\frac{\mathrm{hg}}{2}}=h \sqrt{\mathrm{h}} \\
\text { d'où } \operatorname{lgg}=\sqrt[3]{2 \mathrm{~h}}
\end{gathered}
$$

La formation de la croute glacée à une section déterminée entraine donc un accroissement du niveau d'eau de $25 \%$, pour passer le même débit.

L'augmentation de niveau peut même être plus élevée si l'on tient compte du simple encombrement de la glace; il est donc prudent de tabler sur une augmentation de niveau d'au moins $30 \%$.

Cette augmentation caractéristique du niveau est mise en évidence dans un relevé du Bureau Central Hydrographique de Vienne, representant les variations de niveau du Danube à Fischawend en fonction de la quantité de glace charriée (fig. 1). On observe une brutale augmentation du niveau coïncidant avec toute la durée de prise de la couche superficielle.

L'épaisseur de la couche de glace n'est pas uniforme sur toute la surface; en particulier dans les coudes, il peut $y$ avoir accumulation en certains points et formation de massifs pénétrant assez profondément sous l'eau. Ces conditions peuvent modifier considérablement l'allure des érosions et auront une répercussion sensible sur les fleuves présentant des périodes de prise de longue durée.

\section{Effets sur les barrages et modifications de la ligne de remous}

Considérons un barrage placé en travers d'un fleuve, pour créer une retenue, l'évacuation du débil normal étant assurée par une dérivation, un déversoir, une vanne de fond ou tout autre dispositif. Les glaces charriées seront obligatoirement arrêtées par le barrage, et on aura formation d'une couche progressant vers l'amont. Cette couche de glace crée sur le barrage une poussée qui vient s'ajouter à la poussée hydrostatique. Cette poussée supplémentaire est due à plusieurs causes. On a tout d'abord des forces tangentielles développées par l'eau sur la surlace inférieure de la couche de glace.

En suposant le régime permanent dans le troncon considéré, la pente de la ligne de charge est égale a celle du radier soit i. La force tangentielle à la paroi $\mathscr{E}$, Je rayon hydraulique $R$ h et $i$ sont liés par la relation suivante :

$$
\mathfrak{G}=\mathrm{i} \mathrm{Rh}
$$

Equation qui traduit l'égalité des forces de pesanteur et celle de flottement.

Pour se faire une idée de l'efrort ainsi développé, prenons un cas concret. Je Rhin, en amont de Bâle, présente les caractéristiques suivantes:

- Débit moyen : $\mathrm{Q}=2.000 \mathrm{~m}^{3} / \mathrm{sec}$.

- Pente du fond : $\mathrm{i}=0,00085$.

- Profondeur moyenne $: \mathrm{h}=6 \mathrm{~m} .4$.

- Largeur : $B=145 \mathrm{~m}$.

Après la prise de glace, la profondeur moyenne devient :

$$
\mathrm{hg}=\sqrt[3]{2} \mathrm{~h}==8.10 \mathrm{~m} .
$$

d'où $\mathrm{Rhg}=4 \mathrm{~m} .05$ en négligeant l'action des rives et :

$$
\sigma=\frac{0,00085}{1} \times 4.05=0,0035 \mathrm{~m}
$$

d'ean en mètre courant, ce qui représente pour une largeur de glace de $1 \mathrm{~km}$. une poussée de 3,5 tonnes par mètre de largeur du barrage.

La surface de l'eau fait, en général, un certain angle \% avec l'horizontale. Chaque bloc de glace est

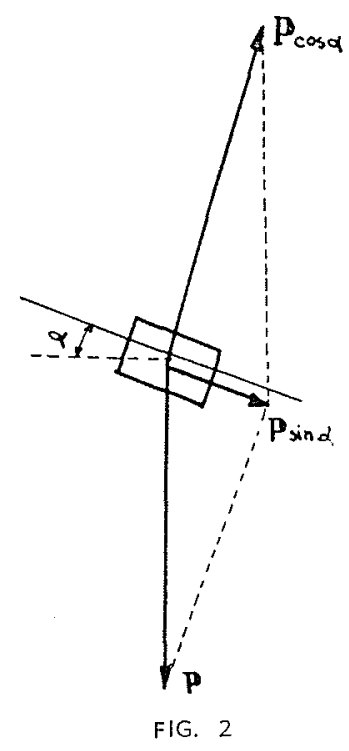

sollicité par son poids $\mathrm{P}$ et par la poussée de l'eau : $\mathrm{P}$ cos anormal à la surface libre. La composante du poids parallèle à surface libre $\mathrm{P} \sin \%$ vient donc 
s'ajouter à la poussée due aux formes du frottement (fig. 2).

Soient p le poids de la couche de glace par metre courant, et mètre de largeur, I la longueur suivant Ie courant, la poussée due au poids de la glace conprise entre les sections 1 el 2 sera:

$$
\text { poussée : } \mathrm{p} \int_{1}^{2} \sin \% \mathrm{~d} \mathrm{l}=\mathrm{p}\left[z_{1}=z_{2}\right]
$$

$z_{1}$ et $z_{2}$ représentant la cote de la surface libre respectivement aux sections 1 et 2 .

Pour l'exemple envisagé, dans le cas d'une couche de glace de $0 \mathrm{~m} .50$ d'épaisseur et pour $1 \mathrm{~km}$. de $10 n-$ gueur, la poussée due au poids sera :

poussée $=\gamma$ glace $0,5,0,85=0,38$ tonne par metre de largeur.

Finalement, la poussée totale pour une longueur de $1 \mathrm{lim}$, sera de $3,88 \mathrm{t} / \mathrm{m}$. peut venir s'ajouter la force tangentielle due au vent agissant sur la partie supérieure de la croûte de slace.

Il arrive aussi que la poussée des glaces sur le; barrages soit considérablement augmentée par le phénomene suivant: In amas de glaces flottantes quasi jointives, s'appuyant sur l'ouvrage de retenue et les rives, possède des interstices d'eau libre; si celle-ci vient à se prendre en glace, elle augmente de volume et il peut en résulter une poussée assez considérable, l'appui sur les rives étant ici un facteur défavorable. C'est à ce genre de phénomène que certains auteurs attribuent l'essentiel de la progression des glaciers. Le rôle essentiel joué par ce type de poussée explique les efforts tentés par les techniciens pour éviter le contact de la glace et du barrage, en envoyant de l'eau du fond au moyen d'émulsion d'air.

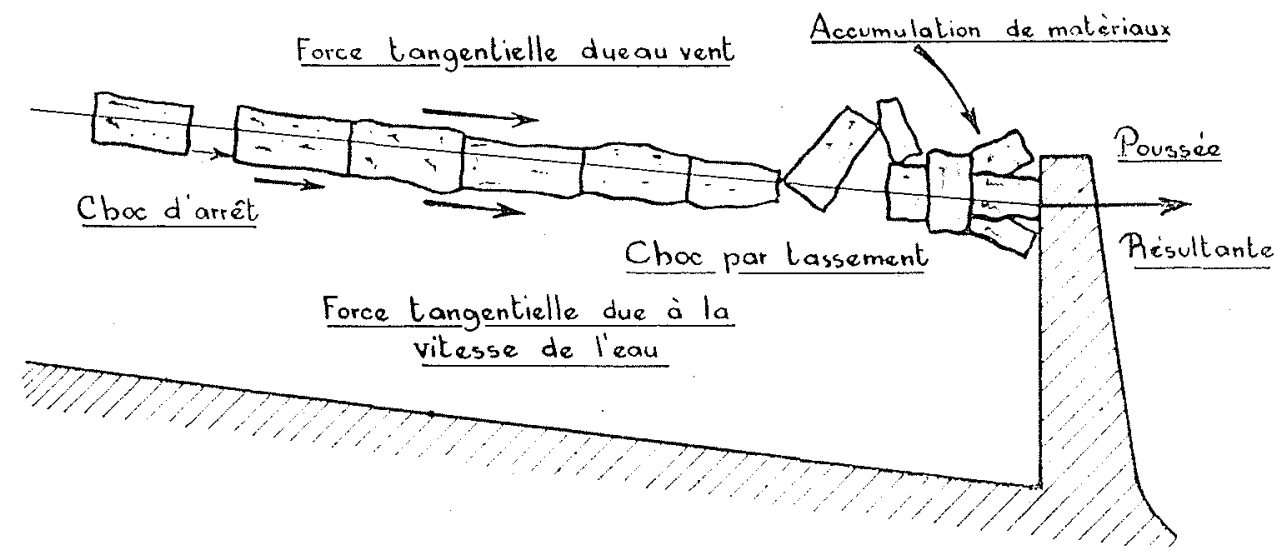

FIG. 3

On obtient aussi un résultat brut; certains effets viennent accroitre la poussée et d'autres la diminuer.

\section{Augmentation de la poussie.}

A la limite de la croûte de glace, les blocs charriés sur le troncon encore libre viennent s'arrêter brulalement sur la conche prise. Il en résulte un choc dont l'effet peut ne pas être négligeable. D'autres choes peuvent prendre naissance dans la croûte de glace elle-même de la manière suivante. Les blocs arrêtés sont enchevêtrés de manière irrégulière; sous l'effet de la pression sans cesse croissante, l'équili'sre de cerlains blocs pent se rompre: il en résulteia un choc violent dont l'action est d'autant plus mirquée que ces chocs peurent se produire au voisinage immédiat du barrage (fig. 3 ).

Enfin aux forces tangentielles dues à l'eau agissant sur la partie inférieure de la couche de glace.
2。 DIMNUTOON DE LA POUSSÉ.

Celte dernière forme de poussée mise à part, les efrorts occasionnés par la glace ont été, dans le paragraphe précédent, supposés entièrement absorbé: par le barrage. Pratiquement les rives supporten! une partie de cette poussée dans une mesure qu'il est difficile d'évaluer (fig. 4). Les rives absorbent une partie d'autant plus grande de la poussée, qu'elles seront plus resserrées et plus tortueuses; au contraire, pour un fleuve large et rectiligne, Ja réaction des rives sera plus faible.

La croûte de glace qui peut être considérée comme fixe par rapport au courant présente, sous l'action de la poussée à laquelle elle est soumise, des déplacements appréciables; on ne possède pas beaucoup de renseignements précis à ce sujet, mais il sembie logique d'admettre que la répartition des vitesses dans une section du fleuve aura, par suite du ralentissement occasionné par les rives, une allure sem- 
blable à celle qu'on observe sur les glaciers, c'est-adire une répartition sensiblement parabolique, avec maximum au milien et vitesse très faible aux bords (expériences du glacier du Rhône, forme des bandes boueuses).

Lat croûte de glace ne conserve pas une épaisseur constante ; elle a tendance à se plisser et à accroître son épaisseur par accumulation de matériaux vers J'aval. En efret, une couche uniforme de glace homo. gène est soumise à des effets de flambement impor-

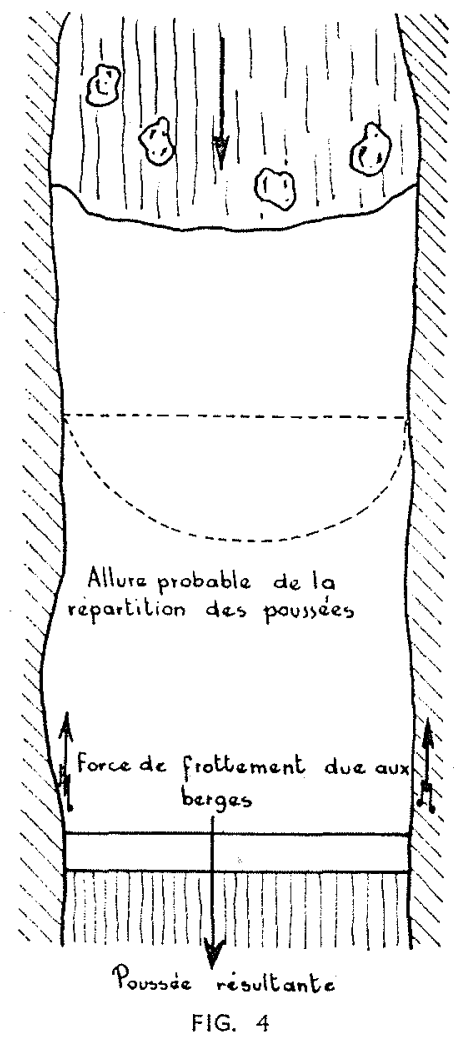

tant qui provoquent des cassures et des plissements. A titre d'indication Harry domne, pour l'effet maximum supportable par une couche de glace de 0,80 d'épaisseur et $200 \mathrm{~m}$. de longueur, une valeur do 0,61 tonne par mètre. Le flambement de la couche glacée ne diminue en rien la valeur de la poussée; au contraire, il occasionne des tassements et des chocs.

On se rend compte aisément que la couche de glace sous l'effet de la poussée a tendance à augmenter d'épaisseur; le poids par unité de surface augmente ainsi que la poussée due au poids, on peut se demander si la croissance simultanée de l'épaisseur et de la poussée aura une limite.

Considérons un tronçon de longueur 1 , subissant la poussée du tronçon amont de longueur L. Soient e l'épaisseur de la glace, b la largeur de la bande considérée, i la pente.

La poussée comprend un terme indépendant de l'épaisseur de ia glace dû au frottement de l'eau ei un terme variant linéairement avec e dû au poids. Si on uéglige les effets sur le troncon I supposé petit par rapport au troncon amont on aura pour la poussece :

$$
F=L b(\sigma+y \text { ie })
$$

Supposons que la croute de glace travaille uniquenrent at flambement. L'effort admissible sera de la forme:

$$
F^{\prime}=K \frac{b e^{3}}{l^{2}}
$$

On atteindra donc une épaisseur fixe quand on aura :

$$
F \leq F^{\prime}
$$

ce qui sera atteint pour une épaisseur critique es. Pour une épaisseur inférieure à e c la couche de glace ne sera pas stable et aura lendance à se déformer et à s'accroître jusqu'à ce que la valeur ee soit atteinte (fig. 5).

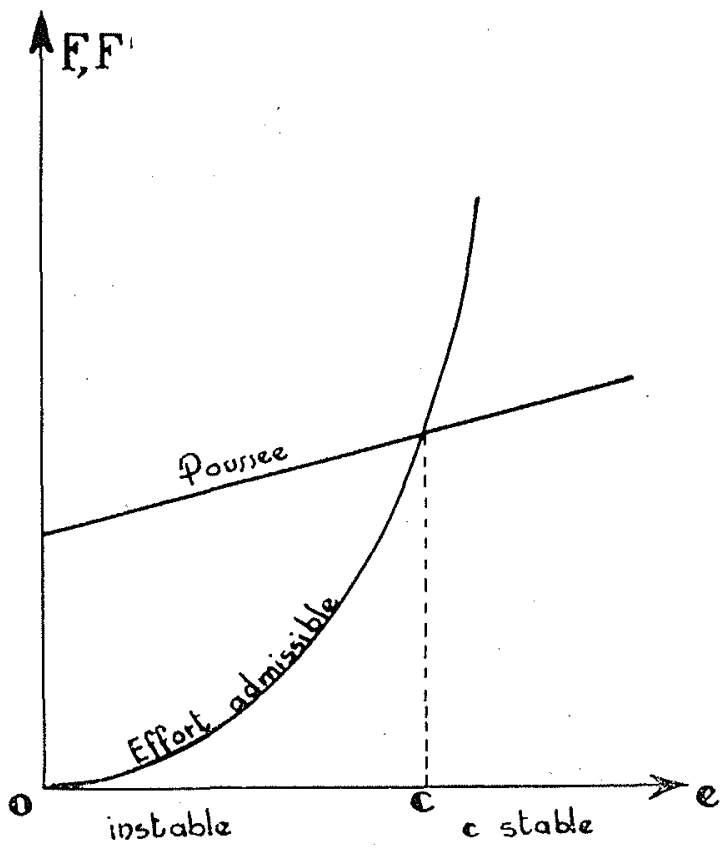

FIG. 5

\section{Effets sur la ligne de remous}

Voyons maintenant comment la présence de la glace va agir sur la forme de la ligne de remous.

Soit u la vitesse moyenne, h la profondeur dans une section, c le coefficient de Chezy, $\frac{\mathrm{d} z}{\mathrm{dx}}$ la pente du fond, $\frac{d h}{d x}$ la pente de surface libre. 


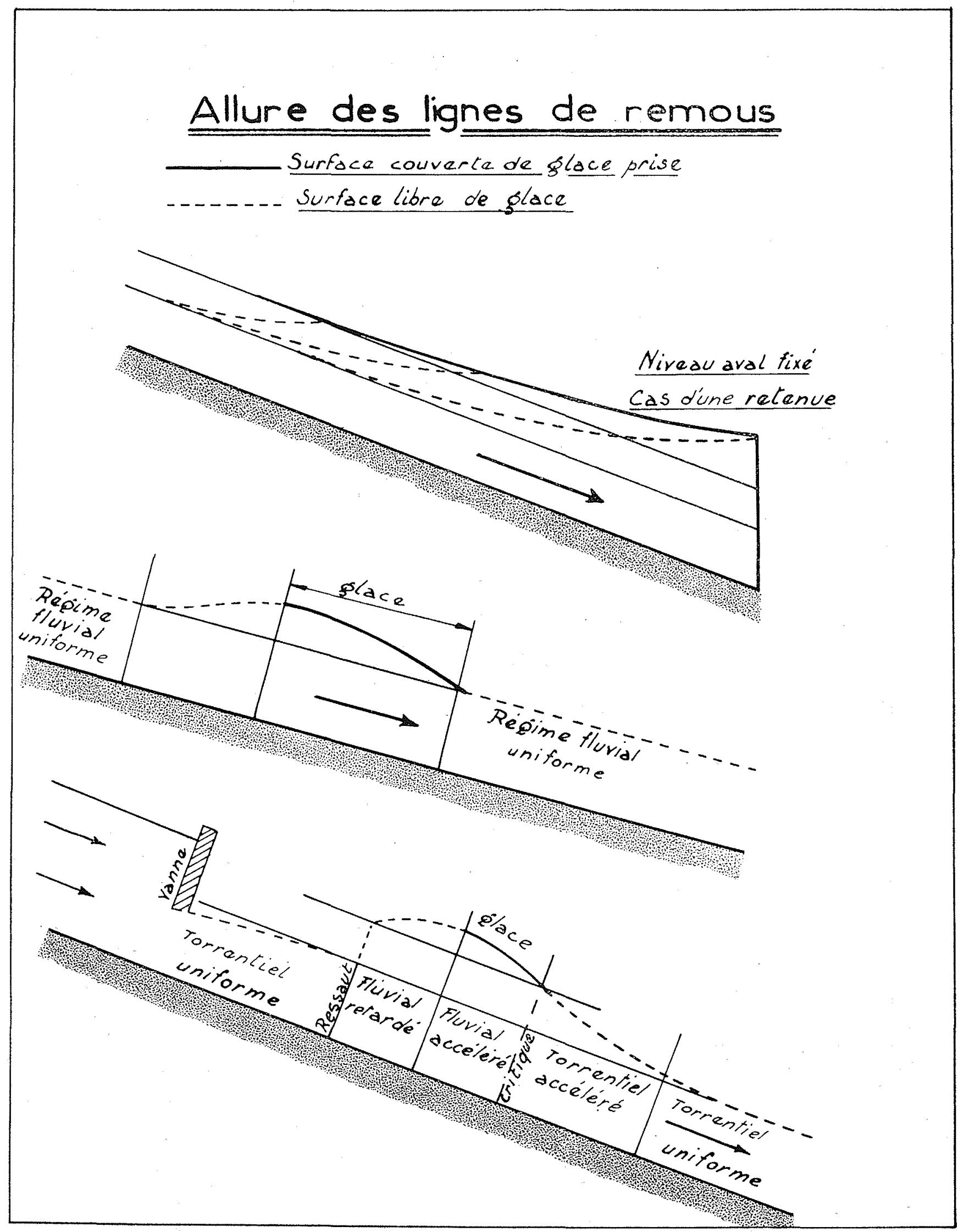


Dans le cas où la surface est libre de glace, on obtient, en appliquant le théorème de Bernouilli à un troncon de longueur $\mathrm{dx}$

$$
\frac{d h}{d x}=\frac{\frac{d z}{d x}-\frac{u^{2}}{c^{2} h}}{1-\frac{u^{2}}{g h}}
$$

dans le cas où la surface est encombrée par une croûte de glace fixe de même rugosité que le fond, on aura :

$$
\frac{\mathrm{dh}}{\mathrm{dx}}=\frac{\frac{\mathrm{d} z}{\mathrm{dx}}-\frac{2 \mathrm{u}^{2}}{\mathrm{c}^{2} \mathrm{l}}}{1-\frac{\mathrm{u}^{2}}{\mathrm{gh}}}
$$

Voyons ce qu'on obtiendra dans le cas du Rhin, cité plus haut, pour une retenue de $12 \mathrm{~m}$. avec $\mathrm{c}=31,5$. de charge passagère, ces singularités ayant fait l'objet d'une étude détaillée dans un précédent article de M. Blanchet, nous n'avons pas jugé nécessaire de nous arrêter sur cette question.

Nous venons de donner un court apercu des effets produits par les glaces sur l'écoulement des fleuves et particulièrement au voisinage des barrages. Si les poussées engendrées sont faibles dans le cas de fortes retenues par suite de la faible valeur des vitesses amont, il n'en sera plus de même pour les retenues de faible hauteur, et en particulier pour les usines au fil de l'eau où le problème revêt toute son importance.

\section{BIBLIOGRAPHIE}

\begin{tabular}{|c|c|c|c|c|c|c|c|c|c|}
\hline $\mathrm{Km}$. vers l'amont. & 0 & 1 & 2 & 3 & 4 & 5 & 6 & 7 & $\infty$ \\
\hline Surface libre... & $\mathrm{h}=12 \mathrm{~m}$ & 11.27 & 10.53 & 4.82 & 9.14 & 8.50 & 7.885 & 7.30 & 6.4 \\
\hline Glace prise & $\mathrm{h}=12 \mathrm{~m}$. & 11.37 & 10.74 & 10.19 & 4.70 & 9.27 & 890 & 8.60 & 8.1 \\
\hline
\end{tabular}

Schoktisch. - Wasserbaü.
Dans la figure 6 sont consignés différents cas possibles de lignes de remous. On reconnaitra aisément les cas classiques d'une retenue dans le premier schéma; les deux autres envisagent les modifi. cations passagères qu'apporte la glace à la ligne de remous d'un canal. La croûte de glaçon joue, par sa rugosité, un rôle analogue à celui créé par une perte
HAnRy. - Action de la glace sur les barrages réservoirs. Rapport da Ve Congrés de l'Union Internationale des Producteur's et Distributeurs d'Energie Electrique.

Jakuschorf, - Uber Grundeis Eisstaungen und Massnahmen zu ihrer Bekämpfung mit. d. Instil. J. Wasserbaü d. T.H. Berlin. 


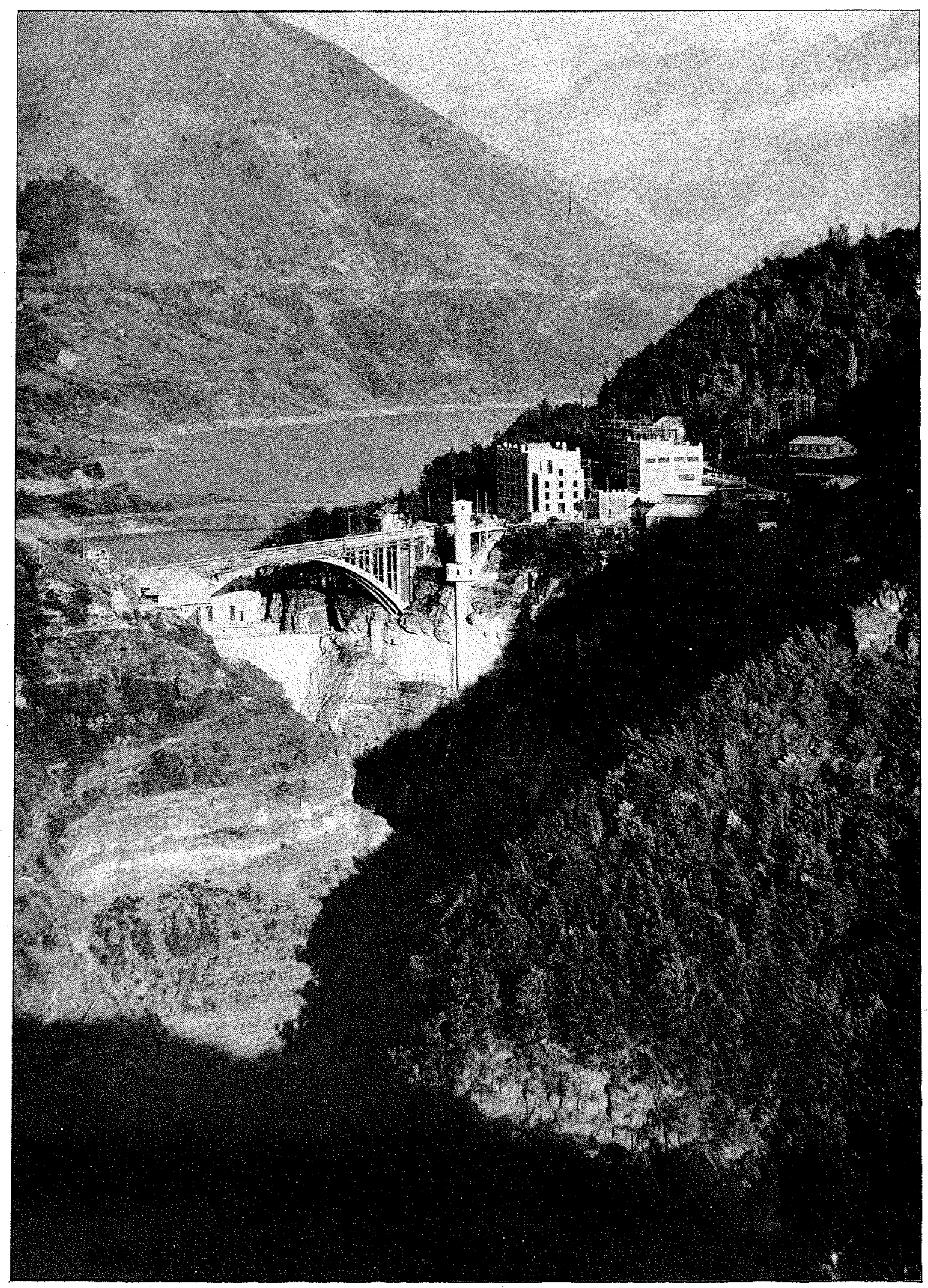

VUE GENERALË DU SAUTET PRISE DAVAL

Photo Bonne et Drac 